\title{
Method for protected noise exposure level assessment under an in-ear hearing protection device: a pilot study
}

Vincent Nadon ${ }^{\mathrm{a}, \mathrm{b}, * *}$, Fabien Bonnet ${ }^{\mathrm{a}, \mathrm{b}}$, Rachel E. Bouserhal ${ }^{\mathrm{a}, \mathrm{b}}$, Antoine Bernier $^{\mathrm{a}, \mathrm{b}}$, Jérémie Voix ${ }^{\mathrm{a}, \mathrm{b}, *}$

${ }^{a}$ Université du Québec, École de technologie supérieure (ÉTS), 1100, Notre-Dame Ouest, Montreal, Quebec, Canada, H3C $1 \mathrm{~K} 3$

${ }^{b}$ Centre for Interdisciplinary Research in Music Media and Technology, 527 Rue Sherbrooke Ouest, Montreal (Qc) Canada, H3A 1 E3

*jeremie.voix@etsmtl.ca

**vincent.nadon@etsmtl.ca 


\section{Keywords}

noise exposure, in-ear noise dosimetry, hearing protection, personal attenuation rating, wearer-induced disturbance 


\begin{abstract}
Objective: To properly measure the effective noise exposure level of workers with hearing protection devices (HPD), the use of in-ear noise dosimeters (IEND) is increasing. Commercial IENDs typically feature one in-ear microphone that captures all noises inside the ear and do not discriminate the residual noise in the earcanal from wearer-induced disturbances (WID) to calculate the in-ear sound pressure levels (SPL). A method to alleviate this particular issue with IENDs and calculate the hearing protection level on-site is therefore proposed. Design: The sound captured by an outer-ear microphone is filtered with the modeled HPD transfer function to estimate the in-ear SPL, this way part of the WIDs mostly captured by the in-ear microphone can be rejected from the SPL. The level of protection provided by the earplugs can then be estimated from the difference between in-ear and outer-ear SPLs. The proposed method is validated by comparing the outcome of the proposed WID rejection method to a reference method. Study sample: The detailed methods are assessed on audio recordings from 16 industrial workers monitored for up to 4 days. Results: The merits of the proposed WID rejection approach are discussed in terms of residual SPL and hearing protection level estimation accuracy. Conclusions: Based on the findings, a method to integrate the proposed WID rejection algorithm in future IENDs is suggested.
\end{abstract}




\section{Introduction}

Despite the efforts to integrate hearing conservation programs in the workplace and the use of HPDs [1], noise-induced hearing loss (NIHL) remains the most common and expensive occupational disease [2]. Although industrial workers do wear hearing protection [1, 3, 4, it is difficult to evaluate the effective noise exposure using a standard sound level meter or a personal dosimeter placed on their shoulder, since the residual sound pressure level (SPL) in the earcanal, that is behind the hearing protector, caused by ambient noise is usually unknown [5]. Indeed, many factors need to be considered when estimating the effective residual noise exposure, such as the attenuation rating of the hearing protector, the quality of the HPD fit [6], the duration the protector was removed during noise exposure [7, 8], as well as how the human auditory mechanisms interact with changes in noise exposure levels [9, 10, 11].

The real-ear attenuation at threshold (REAT) [12] is commonly used to measure the attenuation of the HPD in laboratory based on the hearing thresholds of human subjects with and without HPD. However, the in situ attenuation of the HPD can differ significantly from laboratory values [13, 14] and the REAT can be influenced by the hearing thresholds of tested individuals [15]. For these reasons, objective methods like the field microphone in real ear (F-MIRE) technique were developed to measure the attenuation of HPDs in the field, also referred to as real-world attenuation [14, 16]. The F-MIRE technique uses an outer-ear microphone (OEM) and an in-ear microphone (IEM) to calculate the attenuation of the HPD. Its popularization, and that of other quantitative methods for individual fit testing HPDs also known as Field Attenuation Estimation Systems (FAES) [16, 17], revealed that testing HPD attenuation at regular intervals is important to estimate the real attenuation of the HPD on workers during their work shift. These FAES measurement techniques also feature the calculation of a single number personal attenuation rating (PAR) [18].

Another promising way of assessing adequate protection with HPDs is in-ear noise dosimetry (IEND) [1, 19, 20, 21. IENDs give a more accurate estimate of the residual noise level reaching the eardrum than regular noise dosimeters, which monitor noise levels outside the earcanal [22], because IENDs bypass the need for assumptions and correction factors for microphone placement. In addition, such an approach has also recently proven to

raise awareness among workers as the noise exposure feedback helped them 
reduce their noise dose through more appropriate HPD use [23]. However, the SPL measured inside the earcanal may be affected by various wearerinduced disturbances (WID) 24, such as talking, coughing or chewing, as well as microphonics, which are amplified by the occlusion effect when wearing an earplug [25]. As a result, the earplug can increase the measured in-ear noise dose instead of reducing it. However, middle-ear mechanisms [9, 10], such as the stapedial reflex and nervous system mechanisms [11, are known to be triggered by self-generated noises, such as chewing or speaking. These mechanisms attenuate the intensity with which these self-generated noises ultimately reach the inner ear [10]. Hence, IENDs would benefit from a method to isolate the contribution of such WIDs from the calculated in-ear noise dose to help reflect the real effects of noise exposure levels and duration on the auditory system and better understand why a given individual may seem overexposed based on the SPL measured inside the earcanal [26]. Yet, to this day, commercial IENDs do not feature this option which would be beneficial for future research on occupational hearing loss to properly model the relationship between noise exposure and hearing damage.

To isolate the contribution of WIDs from the in-ear cumulative noise exposure levels, a new approach is proposed in this study using an adaptive filtering method. This method is used for: 1) in-ear dosimetry and 2) as a field attenuation estimation system. To evaluate the method, it is compared to a reference algorithm [26] which uses the coherence variations [26, 27, 28] in the HPD's transfer function, calculated between the OEM and IEM signals, to flag and reject WID noise events. The equivalent noise level $\left(L_{\mathrm{eq}}\right)$ is calculated from the output of both the proposed and reference WID rejection methods with a $3 \mathrm{~dB}$ exchange rate [29] for comparison. In addition, the proposed adaptive filter approach is used to estimate the hearing protection level provided by the HPD during the day. The protection level estimates are validated through comparisons with PAR estimates of a reference method based on cross-spectra and autospectra further detailed in Section 2.2. To estimate the impact of WID rejection on the actual in-ear equivalent SPL, a study is conducted in two industrial environments with 16 human participants exposed to machine noises in their daily routine for up to four days.

The proposed and reference WID rejection methods, as well as a new approach to calculate the PAR are presented in Section 2. Results comparing the reference and proposed algorithms for both PAR calculation and residual SPL estimation after WID rejection are presented in Section 3. Finally, the discussions and conclusions are presented in Section 4 and 5 respectively. 


\section{Methodology}

\subsection{Field data collection}

\subsubsection{Human subjects and tested environments}

To collect sufficient data to compare the WID rejection algorithms presented in this study, 16 participants routinely exposed to noise levels fluctuating between 45 and approximately $100 \mathrm{dBA}$ at their workplaces were selected for tests conducted over up to four days. These participants are divided into two groups. The first group, hereafter called Noise Group 1 (NG1), consists of 3 individuals working in a Computer Numerical Control (CNC) machining workshop and aged between 36 and 44 years old. The second group, hereafter called Noise Group 2 (NG2), is formed by 13 individuals in a woodworking school and aged between 18 and 44 years old, with a median age of 28 . In their daily routine, NG1 participants were mainly exposed to background ventilation noise as well as the noise of machining metal parts coming from a CNC machine behind a window. NG2 participants were exposed to background ventilation noise as well as a variety of woodworking machinery noises produced, for example, by industrial wood jointers, planers and wood hammers. Therefore, NG2 participants were almost constantly exposed to noise for a maximum period of 5 hours, with a 30-minute lunch break for some, while NG1 participants were exposed more occasionally to loud noises as they entered and left the workshop during their 8-hour work shift. The approximate duration of the experiment per day is presented in Table 1. All participants were indoors in semi-reverberant rooms during the tests, thus eliminating other possible outdoor noise sources from wind, traffic, etc. The experiment with NG1 participants was approved by the Comité d'éthique pour la recherche, the internal review board (IRB) of the École de technologie supérieure. The participation of NG2 subjects was approved by the IRB of the woodworking school.

\subsubsection{Audio recording equipment}

The raw audio data was recorded on-site with a recording device, dubbed the Auditory Research Platform (ARP), developed over the years at the NSERC-EERS Industrial Research Chair in In-Ear Technologies [30, and shown in Fig. 1. This portable device specifically designed to perform measurements in high noise level environments features two high attenuation earplugs, each equipped with electronic components to perform the acoustical measurements: an IEM, an OEM and two miniature loudspeakers. A 
Table 1: Approximate duration of the experiment per day for each group of participants, numbered from $\# 1$ to $\# 16$ for a total of approximately 294 hours of recorded data, of which a subset of approximately 147 hours are used in the current study due to reasons detailed in Section 2.4.1

\begin{tabular}{|c|c|c|c|}
\hline Group & Participants \# & Days & Hours/day \\
\hline NG1 & $1-3$ & 4 & 8 \\
NG2 & $4-7$ & 4 & 5 \\
NG2 & $8-10,12-14,16$ & 4 & 6 \\
NG2 & 11 & 2 & 6 \\
NG2 & 15 & 1 & 6 \\
\hline
\end{tabular}

fit-test was performed before entering the noisy environment to ensure a proper fit quality and sufficient protection [31. The participants were wearing the portable device in a fanny pack for easier transportation, and the earpieces' wires were covered by their aprons or shirts and attached to their collar using a shirt clip to reduce the risk of microphonics and to ensure the wires would not hang out and risk getting stuck in machinery as shown on the right in Fig. 1. To verify the accuracy of the ambient SPLs measured with the designed portable system, the participants were also wearing a Spark 706RC reference dosimeter (Larson Davis, NY, USA) to measure the ambient SPL $L_{D}$ (see Table 2 for definition of the variables used further on). The dosimeter's microphone was attached to their shoulder on the same side as the ear being tested.

\subsection{Personal attenuation rating}

The PAR is a single number estimate that quantifies the noise attenuation achieved by an HPD on a given individual [18]. A low PAR resulting from poor HPD fit can explain why a worker was overexposed.

In this study, the PAR is estimated using two different methods. The first method uses the passive attenuation of the earplug based on the $H_{3}[n]$ transfer function, shown in Fig. 2, estimated with the average of the ratios of the cross-spectra and autospectra of OEM and IEM signals [27. The $\mathrm{PAR}_{\text {ref }}$ is then calculated with this earplug attenuation per octave band. The second method uses the difference between $L_{\text {eq, OEM }}$ and the residual SPL, denoted $L_{\mathrm{AF}}$, estimated from the adaptive filter output, where the adaptive filter identifies the transfer function of the earplug's passive attenuation as 

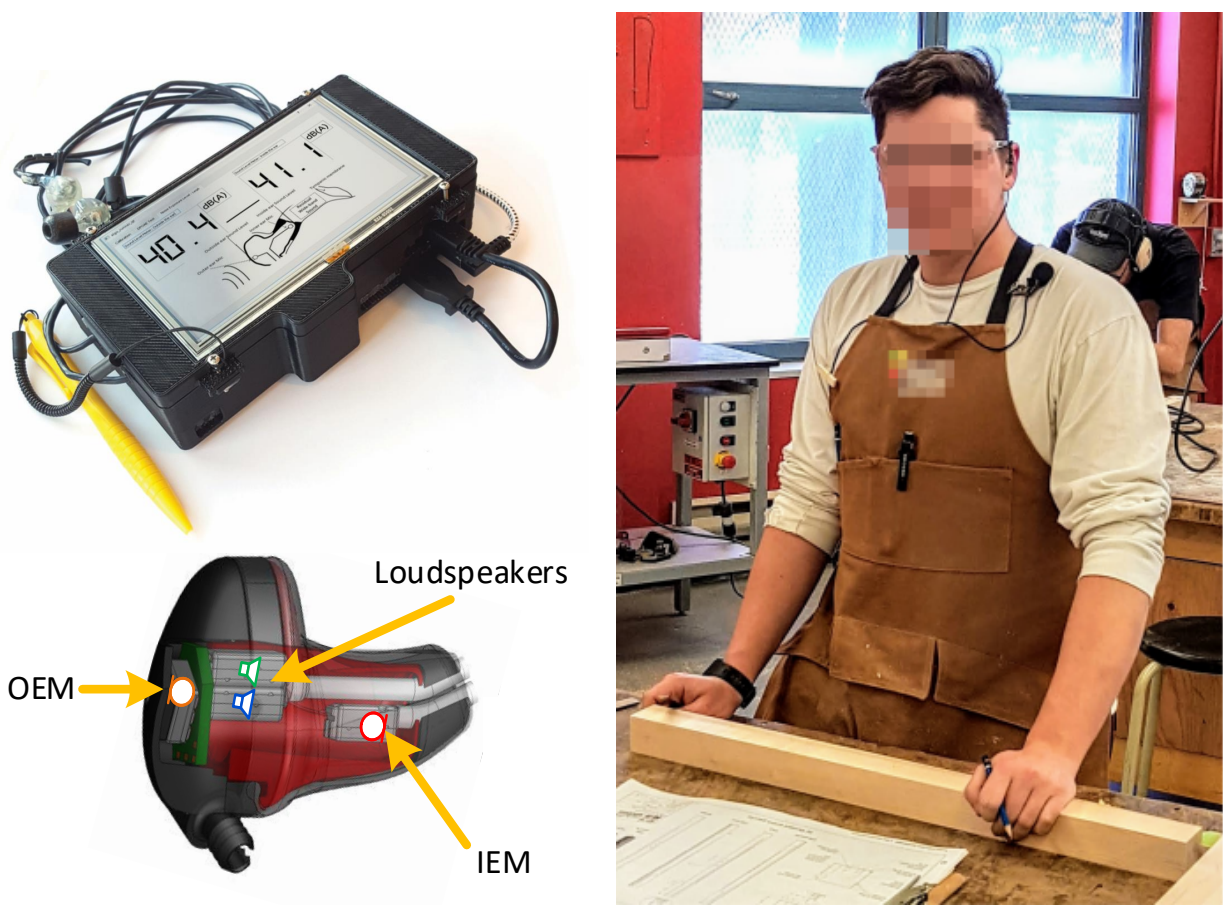

Figure 1: The Auditory Research Platform (ARP) with hearing protection earpieces including electronic components for outer-ear and in-ear noise dosimetry (left), and a worker wearing the ARP during in situ measurements (right) 
detailed in Section 2.3. This method is developed and used in this study as it performs a less computationally exhaustive PAR assessment technique, since the identification of the transfer function with Fast Fourier Transform (FFT) is no longer needed. This gives the possibility to calculate the PAR and assess the fit quality for the whole duration that the protector is worn, whereas current FAES generally measure the PAR only once, before being exposed to noise. The proposed PAR $\mathrm{AF}_{\mathrm{A}}$ is calculated as follows:

$$
\mathrm{PAR}_{\mathrm{AF}}=L_{\mathrm{eq}, \mathrm{OEM}}-L_{\mathrm{AF}}
$$

where the $L_{\text {eq,OEM }}$ is the equivalent sound level calculated from the A-weighted OEM signal and $L_{\mathrm{AF}}$ is the A-weighted level of the adaptive filter output further explained in Section 2.3 .

The PAR $\mathrm{PF}_{\mathrm{A}}$ estimates are then compared with PAR $\mathrm{P}_{\text {ef }}$ to ensure that they give similar results so that eventually the PAR $\mathrm{AF}_{\mathrm{F}}$ could be integrated in a portable IEND and provide information on hearing protection level to the user. In this work, the PAR can also be used to detect when the earplug transfer function is not correctly identified with the adaptive filter; on the assumption that a) the passive attenuation of a given HPD should not vary over a short period of time if the earplug is fit correctly, and b) the estimation of the PAR should also remain constant and positive over a couple seconds. The continuous estimation of the PAR can therefore be used to reject incorrect estimates of the residual SPL when using the proposed WID rejection method later detailed in Section 2.3

\subsection{Proposed residual sound pressure level estimation using adaptive filtering}

WIDs are more present in the IEM signal $i(n)$ than in the OEM due to the occlusion effect and the attenuation of the earplug. Hence, in order to reject the WIDs from the in-ear residual SPL, the OEM signal denoted $o(n)$, is used to estimate the residual SPL behind the earplug by filtering this signal with an estimate of the earplug transfer function $\hat{H}$ to obtain $i^{*}(n)$.

To estimate the earplug transfer function $\hat{H}$, the broadband ambient noise is captured using the OEM and IEM while the earpiece is worn. The earplug transfer function is then modeled continuously by a finite impulse response (FIR) filter obtained using an adaptive Normalized Least-Mean 
Square (NLMS) method as follows:

$$
\begin{aligned}
i^{*}(n) & =\mathbf{w}^{T}(n) \mathbf{o}(n), \\
e(n) & =i(n)-i^{*}(n), \\
\mathbf{w}(n+1) & =\mathbf{w}(n)+\frac{\mu e(n) \mathbf{o}(n)}{\mathbf{o}^{T}(n) \mathbf{o}(n)}
\end{aligned}
$$

where $o(n)$ is the OEM signal and $i(n)$ the IEM signal. The filter coefficients $\mathbf{w}(\mathbf{n})$ converge towards the earplug transfer function by minimizing the error $e(n)$. The $\mu$ adaptation step size is set to 0.5 , based on a previous study [32], to correctly identify the transfer function within two seconds with an adaptive filter of $N=200$ coefficients. The filtered output is then used as the in-ear sound pressure estimate $i^{*}(n)$, as shown in Fig. 2. This signal is then passed through an A-weighted filter and used to calculate the equivalent sound level $L_{\mathrm{AF}}$ in $\mathrm{dBA}$.

In order to obtain a precise estimate of the earplug transfer function and ensure proper estimation of $\mathrm{PAR}_{\mathrm{AF}}$ and $L_{\mathrm{AF}}$, an adaptation time of approximately 10 seconds is used. However, when a WID is present within the $0.33 \mathrm{~s}$ period of analysis during the adaptation of the filter and the ambient SPL is too low, the identified earplug transfer function is incorrect and results in a negative PAR. Part of the results obtained with an inadequate earplug transfer function estimate resulting from a WID are removed by using $L_{\mathrm{AF}}$ in the daily $L_{\mathrm{eq}}$ only when a valid PAR $\mathrm{PF}$ is estimated on 30 seconds, i.e. when $\mathrm{PAR}_{\mathrm{AF}}>0 \mathrm{~dB}$. This criterion reduces the risk of overestimating $L_{\mathrm{AF}}$ past the 10-second adaptation period.

\subsection{Reference WID detection and rejection method}

The reference WID detection method is based on the calculation of the coherence between the IEM and OEM signals [28]. The method is based on the fact that when the sound pressure level measured inside the ear is mostly due to surrounding external noise, a high coherence is observed between the two microphone signals as sound travels predominantly through the earplug. When the IEM is disturbed by WIDs, such as speech, this coherence decreases within the frequency range of the disturbance signal. When such coherence is measured at regular time intervals and averaged across a predefined frequency range $\left[f_{\min } f_{\max }\right]$, it is then possible to define an indicator, here referred to as $\Delta(\mathrm{dB})$, to detect WID events [24, 26]. A binary decision is taken by comparing this indicator to a predefined threshold parameter 
Table 2: Definition of $L_{\text {eq }}$ and PAR variables

\begin{tabular}{|l|l|}
\hline Variable & Definition \\
\hline$L_{\mathrm{AF}}$ & $\begin{array}{l}\text { In-ear } L_{\mathrm{eq}} \text { calculated with the } i^{*}(n) \text { output of the } \\
\text { adaptive filter ('AF') }\end{array}$ \\
\hline$L_{\mathrm{TF}}$ & $\begin{array}{l}\text { In-ear } L_{\mathrm{eq}} \text { calculated from the } i(n) \text { signal where the } \\
\text { noise contribution of WIDs are excluded using the coher- } \\
\text { ence from the transfer function-based reference method } \\
(\text { 'TF') }\end{array}$ \\
\hline$L_{D}$ & $\begin{array}{l}\text { Ambient } L_{\mathrm{eq}} \text { calculated from the Larson Davis reference } \\
\text { dosimeter with shoulder microphone }\end{array}$ \\
\hline PAR $_{\mathrm{ref}}$ & $\begin{array}{l}\text { personal attenuation rating estimated from the } \\
\text { reference ('ref') method that uses the average of the } \\
\text { autospectrum and cross-spectrum estimates of the } i(n) \\
\text { and } o(n) \text { signals. }\end{array}$ \\
\hline PAR $_{\mathrm{AF}}$ & $\begin{array}{l}\text { personal attenuation rating estimated using the } L_{\mathrm{eq}} \text { cal- } \\
\text { culated from the estimated } i^{*}(n) \text { adaptive filter ('AF') } \\
\text { output and the } L_{\mathrm{eq}, \text { OEM }}\end{array}$ \\
\hline
\end{tabular}

$\Delta_{t h}$ above which it is assumed that WIDs are present and contributing to the noise level measured by the IEM. The $L_{t h}$ threshold level is selected to distinguish between high-level WIDs -like shouted speech- which have a substantial impact on the noise levels measured by the OEM, and low-level WIDs -like chewing- which should only affect the signal measured by the IEM. To estimate the in-ear noise dose while excluding the noise contribution from low-level WIDs, the earplug transfer function is subtracted from the noise level measured by the OEM. For high-level WIDs, the noise level at the IEM is assumed to be the same as the last recorded audio sample where no WIDs are detected [26, 24]. This approach isolates most of the noise contributions from the wearer, regardless of the WID's type [33, 24]. In the present work, the parameter values of $\Delta_{t h}=0.75$ found by [24] is used, but the SPL threshold $L_{t h}$ is set at $70 \mathrm{~dB}$ as recommended by a more recent study [33].

\subsubsection{Artifact rejection in recorded audio signals}

Due to a technical issue in the ARP software developed for this study, short discontinuities in the audio data recorded were found at random times, 


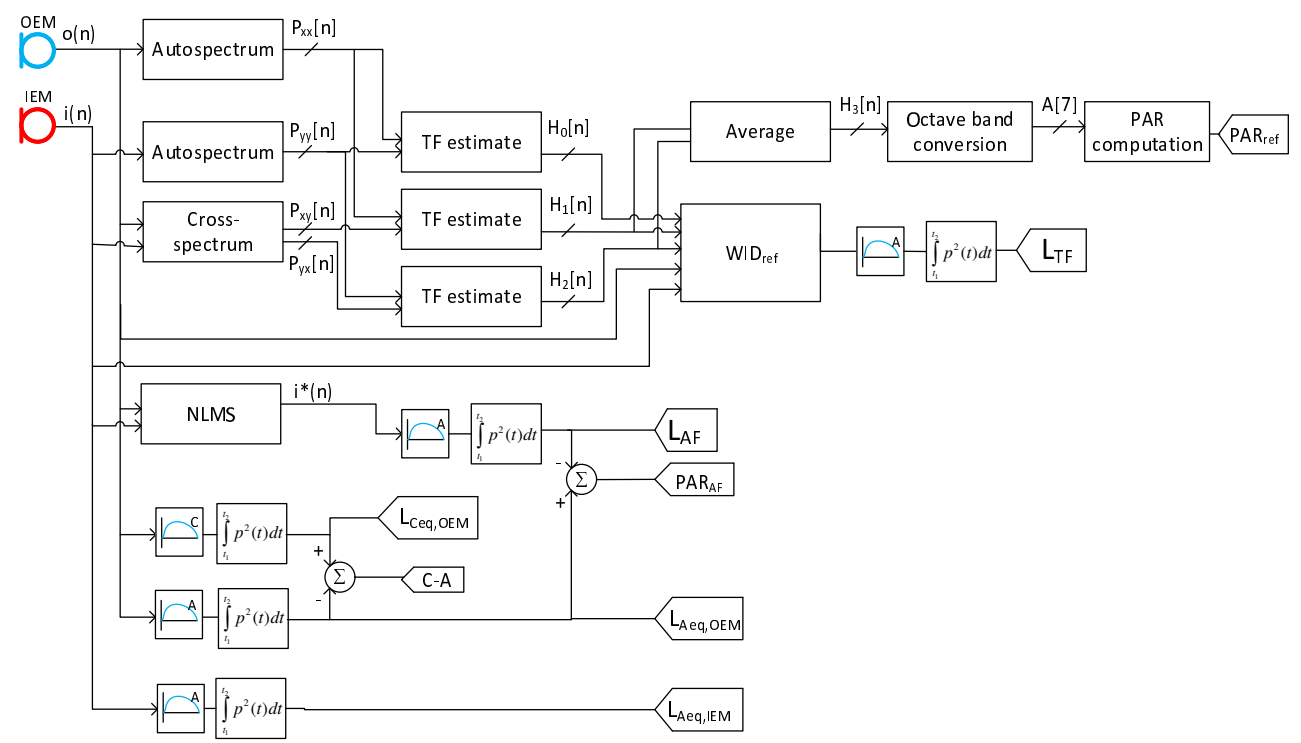

Figure 2: Schematic of the algorithm used to estimate the equivalent noise levels defined in Table 2 and other noise metrics 
simultaneously on all audio channels. Such discontinuities were thoroughly investigated and attributed to a lack of proper interruption management resulting in overwriting part of the audio circular buffers. Since these discontinuities happen randomly but were in-sync on all acquired signals, partial recovery was possible and the in situ collected data was judged to be of sufficiently high-value to justify the development of an artifact rejection algorithm.

In order to clean the recorded audio data and remove the discontinuity artifacts, a comparison is made between the low-frequency coherence $(\Delta)$ and a higher-frequency coherence indicator $\left(\Delta_{H F}\right)$ defined here, the only difference between $\Delta_{H F}$ and $\Delta$ being that $\Delta_{H F}$ is calculated among thirdoctave frequencies from 1500 to $8000 \mathrm{~Hz}$ instead of 200 to $1250 \mathrm{~Hz}$. The audio signal is discarded from post-processing whenever $\Delta_{H F}$ exceeded $\Delta$ by at least $3 \mathrm{~dB}$, during the $0.33 \mathrm{~s}$ period of analysis. This allowed rejecting the discontinuity artifacts while retaining the rest of the signal. In most situations, WIDs are not rejected by this approach as WIDs contribute mostly to lower frequencies [26], hence affecting $\Delta$ more than $\Delta_{H F}$. An example of the proposed data correction is shown in Fig. 3 where the audio signals with the discontinuities recorded on a Head and Torso Simulator (HATS) are effectively rejected by the presented coherence-based approach. This artifact rejection algorithm is used to remove all discontinuities from the processed audio before the analysis presented in this study, which resulted in the removal of approximately half of the total useful audio data, but still retaining close to $147 \mathrm{~h}$ of reliable in situ recordings.

\section{Experimental results}

\subsection{Estimation of the PAR}

To validate the proposed PAR $\mathrm{AF}$, it is compared to PAR $\mathrm{P}_{\text {ref }}$ in the BlandAltman plot in Fig. 4. Such Bland-Altman plots are generally used to analyze the agreement between two different methods. As shown in the figure, the difference between the methods ranges from -8.3 to $6.6 \mathrm{~dB}$ at the $2.5^{\text {th }}$ and $97.5^{\text {th }}$ percentile (right graph).

\subsection{Estimation of the residual sound pressure level}

To calculate the difference between the estimated residual SPL and the original $L_{\text {eq,IEM }}$, the OEM and IEM sound pressure levels of each individual

participant are first calculated over $0.33 \mathrm{~s}$ and then accumulated each one 


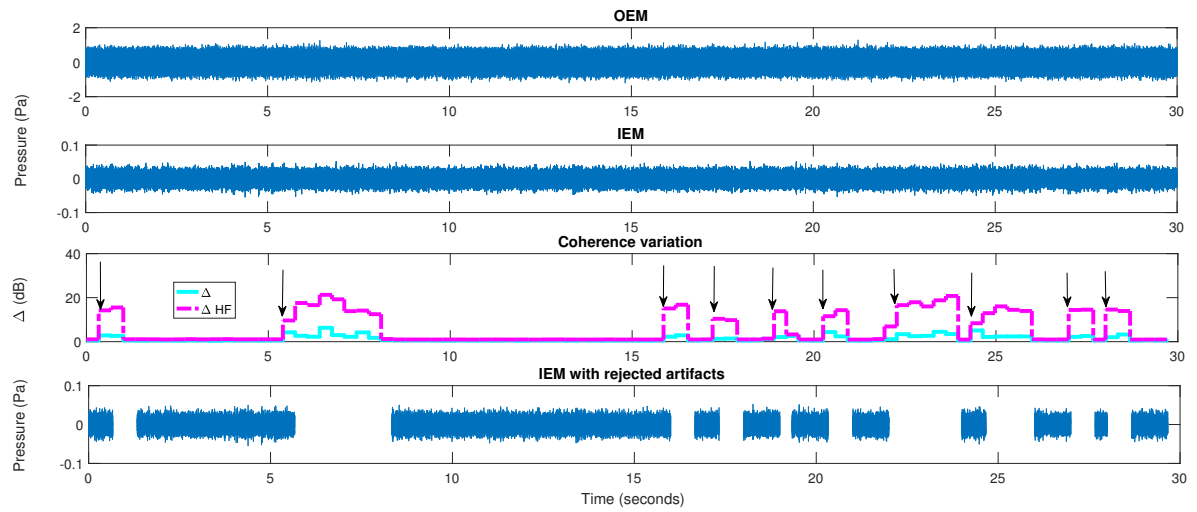

Figure 3: OEM and IEM signals, where no WIDs are present when the system is tested on a HATS. Discontinuities are not visible at first sight but caused erroneous values in the $\Delta$ The discontinuities are well detected, as pointed by the arrows, by comparing $\Delta_{H F}$ with $\Delta$ and are rejected both from the OEM and IEM signals (only the IEM is shown in the figure above)
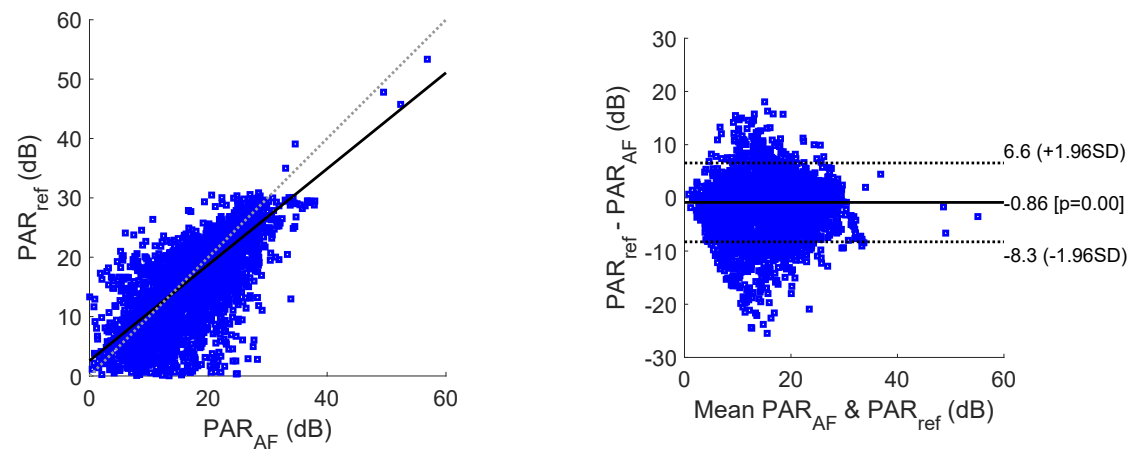

Figure 4: Bland-Altman plots showing the correlation of the $\mathrm{PAR}_{\mathrm{AF}}$ approach and the reference $\mathrm{PAR}_{\text {ref }}$ approach used as a benchmark for all participants on all tested days (left) and the difference between the methods in function of the mean value of both methods (right) 
second. As an example, these SPLs are shown at the top of Fig. 5a for one participant. These one-second equivalent sound pressure levels are compared between the different approaches $\left(L_{\mathrm{AF}}, L_{\mathrm{TF}}, L_{\mathrm{eq}, \mathrm{IEM}}\right.$ and $\left.L_{\mathrm{eq}, \mathrm{OEM}}\right)$ and accumulated over 30 seconds, shown at the $L_{\text {eq,30s }}$ mark at the bottom of Fig. 5 a. These $L_{\text {eq }, 30 \text { s }}$ levels are then shown over a 10-minute period in Fig. $5 b$ for

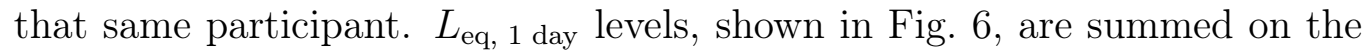
number of valid $L_{\text {eq, }}$ 10min data points for the work day. The graph in Fig. 5 a shows the typical good agreement observed between $L_{\mathrm{AF}}$ and $L_{\mathrm{TF}}$. It also illustrates the reduction in sound pressure level achieved with both methods rejecting WIDs when an accurate earplug transfer function is estimated. Fig. 6 6 shows that there are negligible differences between $L_{D}$ and $L_{\text {eq, OEM }}$.

Results shown in Fig. 5 \& 6 are illustrative of a typical measurement day and are further described to ease the interpretation of the results: during the experiment the participant may talk during low ambient noise levels, as shown with the higher $L_{\text {eq,IEM }}$ levels at the 0 and 10-minute mark in Fig. 6. Such WIDs can occur at any moment during the 30 -second period of analysis and causes the earplug transfer function to be incorrectly identified, thus resulting in a low $L_{\mathrm{AF}}$ level. On the other hand, an incorrect transfer function may also be identified when the participant generates a low-level WID during the 10 seconds used for the filter adaptation for $L_{\mathrm{AF}}$, which occurs every 30 seconds in this study. In this case the identified transfer function overestimates the residual SPL $L_{\mathrm{AF}}$ when compared to $L_{\mathrm{eq}, \mathrm{IEM}}$ as shown at 20,30 , 100 and 200 minutes in Fig. 6. The variations in transfer function estimates are also visible in $\mathrm{PAR}_{\mathrm{AF}}$ in Fig. $5 \mathrm{~b}$ at approximately 106.3 minutes when the participant generated a WID, rejected by the reference method, but causing an incorrect filter adaptation for the proposed method. The PAR also decreases past 107.3 minutes due to the ambient noise level being too low, compared to the physiological noise behind the earplug, to correctly estimate the earplug transfer function. In these situations, the proposed method could benefit from a more robust PAR criterion than $\mathrm{PAR}_{\mathrm{AF}}>0 \mathrm{~dB}$. This criterion must be optimized to better discriminate such artifacts and reject $L_{\mathrm{AF}}$ when the earplug transfer function is no longer adequate.

High-level disturbances are detected at 15,18 to 20 and 27 to 28 seconds in the IEM signal in Fig. 5a, which are rejected in both $L_{\mathrm{TF}}$ and $L_{\mathrm{AF}}$. In the illustrated case, these disturbances come from throat clearing, but also from audio notifications of the system triggered through participant interactions with the measurement software (e.g. waking up the device).

To compare the performance of the proposed algorithm $L_{\mathrm{AF}}$ with the 


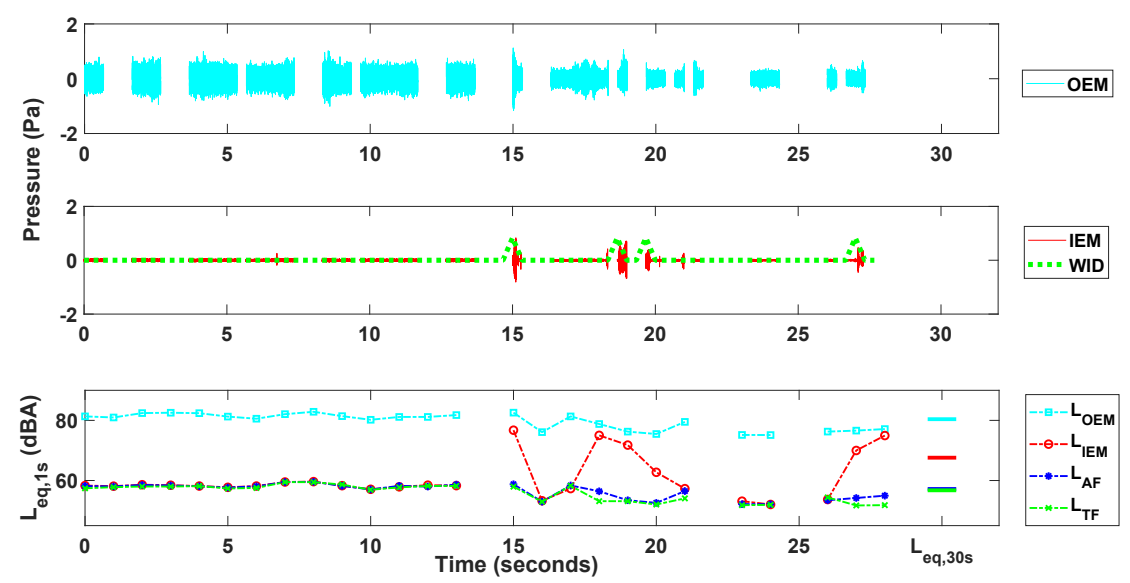

(a)

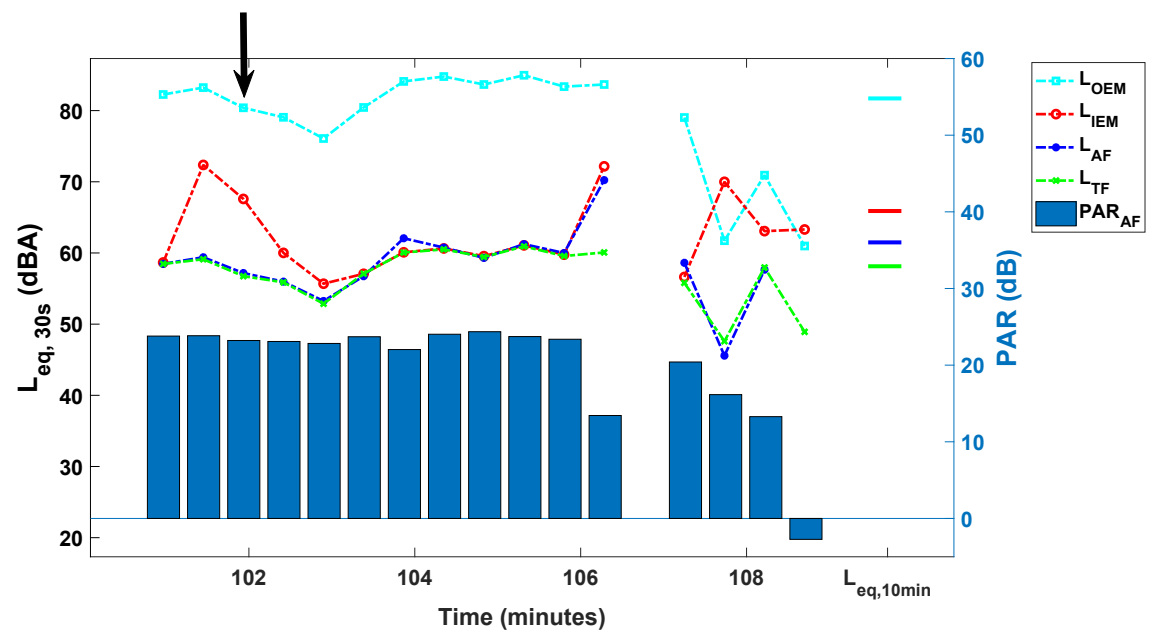

(b)

Figure 5: Example of typical cumulative equivalent noise levels for an individual. The figures show different steps of analysis: (a) over $30 \mathrm{~s}$ with the sound pressure expressed in Pascals at the top, (b) over 10 min and showing as well the proposed PAR estimate every $30 \mathrm{~s}$. The accumulated $L_{\text {eq }}$ levels for each microphone and different methods for the residual SPL are shown at the right end of each plot. In (a) the WID detection flags of the reference algorithm indicate when the high level WID events have been isolated from the IEM signal to calculate $L_{\mathrm{TF}}$. The arrow in (b) indicates the index of the 30 seconds of plot (a). The blank parts in waveform in (a) are the audio data rejected with the artifact rejection algorithm, whereas in (b) the $107^{\text {th }}$ minute is rejected due to the presence of stimuli generated to measure otoacoustic emissions as part of a follow-up study 
reference WID rejection method $L_{\mathrm{TF}}$, the 1-day residual SPLs after WID rejection are compared to $L_{\text {eq,IEM }}$. These comparisons are displayed as a distribution for the different measurements $\left(L_{\mathrm{AF}}, L_{\mathrm{TF}}\right.$ and $\left.L_{\mathrm{D}}\right)$ in the normal probability plot of Fig. 7. As shown in this figure, the intersection of the plots with the 0.5 probability, i.e. the mean difference, is at $-3.4 \mathrm{~dB}$ for $L_{\mathrm{AF}}$ and $-4.8 \mathrm{~dB}$ for $L_{\mathrm{TF}}$. The proposed and reference WID rejection methods resulted in a similar $(p>0.05$, paired $t$-test $)$ mean difference, with approximately $0.3 \mathrm{~dB}$ overall between the two methods. The comparison of $L_{\mathrm{D}}$ with $L_{\text {eq,IEM }}$, ranging from -7.9 up to $22.7 \mathrm{~dB}$ with an average of $9.4 \mathrm{~dB}$, shows the difference between the estimation of an individual's noise exposure level measured at the shoulder and an in-ear microphone behind an earplug. These differences account for the free-field to occluded earcanal correction [34, the attenuation provided by the earplug, and physiological noises measured exclusively by the IEM.

\section{Discussion}

\section{1. $P A R$}

The proposed $\mathrm{PAR}_{\mathrm{AF}}$ between the $i^{*}(n)$ and $o(n)$ signals gives similar results to $\mathrm{PAR}_{\text {ref }}$ in most cases. However, large differences of approximately -8.3 and $6.6 \mathrm{~dB}$, as shown in the Bland-Altman plots in Fig. 4, are caused due to the presence of WIDs during adaptation of the filter or the calculation of $\mathrm{PAR}_{\text {ref. }}$. Whereas the $6.6 \mathrm{~dB}$ difference is explained by incorrect earplug transfer function estimate, thus underestimating $\mathrm{PAR}_{\mathrm{AF}}$. Based on these assumptions, $\mathrm{PAR}_{\text {ref }}$ is expected to differ slightly from $\mathrm{PAR}_{\mathrm{AF}}$ resulting in the correlation coefficient of $R^{2}=0.6$ (left graph). Such differences are acceptable for these in situ measurements considering the test conditions were not controlled, and could be improved by adapting the filter for the earplug transfer function and measuring $\mathrm{PAR}_{\text {ref }}$ at an optimal moment. Despite these possible improvements in PAR estimates, the proposed algorithm's PAR $_{\mathrm{AF}}$ did go up to approximately $30 \mathrm{~dB}$, as shown on the left of Fig, 4 , and such high PAR suggests that the participants were adequately protected during their work with the foam eartips provided with the designed earpieces.

To improve the earplug transfer function identification by avoiding filter adaptation when the PAR is low, the current PAR criterion of $\mathrm{PAR}_{\mathrm{AF}}>$ $0 \mathrm{~dB}$ should be adjusted. For instance, the PAR criterion could be a function of the earplug fit quality in various situations, i.e. a criterion based on a 
threshold discriminating a good fit from a bad fit. For example, this criterion could be set at $25 \%$ below the PAR measured in an anechoic chamber. An adequate PAR criterion would also improve the proposed method's $L_{\mathrm{AF}}$ estimate. Moreover, the PAR should be estimated in high ambient noise settings when participants remain silent and do not generate WIDs. Following such improvements, the proposed PAR $\mathrm{AF}_{\mathrm{F}}$ could be used to estimate the protection level of workers in real time.

\subsection{Residual sound pressure level}

According to the results shown in Fig. 7 the mean difference between $L_{\text {eq,IEM }}$ and $L_{\mathrm{AF}}$, for all participants over separate days, is approximately $-3.4 \mathrm{~dB}$ for $L_{\text {eq }, 1 \text { day. The }} 1.4 \mathrm{~dB}$ difference between the proposed $(-3.4 \mathrm{~dB})$ and reference $(-4.8 \mathrm{~dB})$ WID rejection methods, is potentially caused by either: A) the adaptation of the filter during a WID which results in an incorrect transfer function estimate which also reduces the PAR, as shown for instance in Fig. $5 \mathrm{~b}$ at the 106.3-minute mark; B) the reference method rejected more high-level WIDs during low ambient SPL than the proposed method due to the influence of these WIDs on the OEM signal which is used with the adaptive filter to estimate the residual SPL; or C) abrupt increases in the ambient SPL occurred during high-level WIDs, causing the reference algorithm to underestimate the in-ear noise exposure compared to the proposed algorithm. This hypothesis in C) is attributed to the reference algorithm using the last $L_{\text {eq }}$ measured prior to WID detection [26], whereas the proposed method relies on the adaptive filter output which might be a better representation of the ambient SPL variations that occurred during high-level WIDs.

According to the experimental results, the proposed adaptive filter method appears as an efficient way to reject the WID noise events from the measured in-ear $L_{\text {eq }}$. When a proper earplug attenuation transfer function is estimated, as shown with $\mathrm{PAR}_{\mathrm{AF}}$ higher than $23 \mathrm{~dB}$ in Fig. 5b, the proposed adaptive filtering method leads to lower residual SPL by rejecting the WIDs. As previously discussed, the comparison with the reference WID rejection method (Fig. 6 \& 7) shows that a more efficient WID rejection is nonetheless possible by improving the proposed method to reduce the impact of high-level WIDs

captured by the OEM and avoid overestimating the residual SPLs because of improper earplug transfer function estimates. The distinction between lowlevel and high-level WIDs is not present in the proposed approach, which uses $o(n)$ combined with the earplug's transfer function identified through 


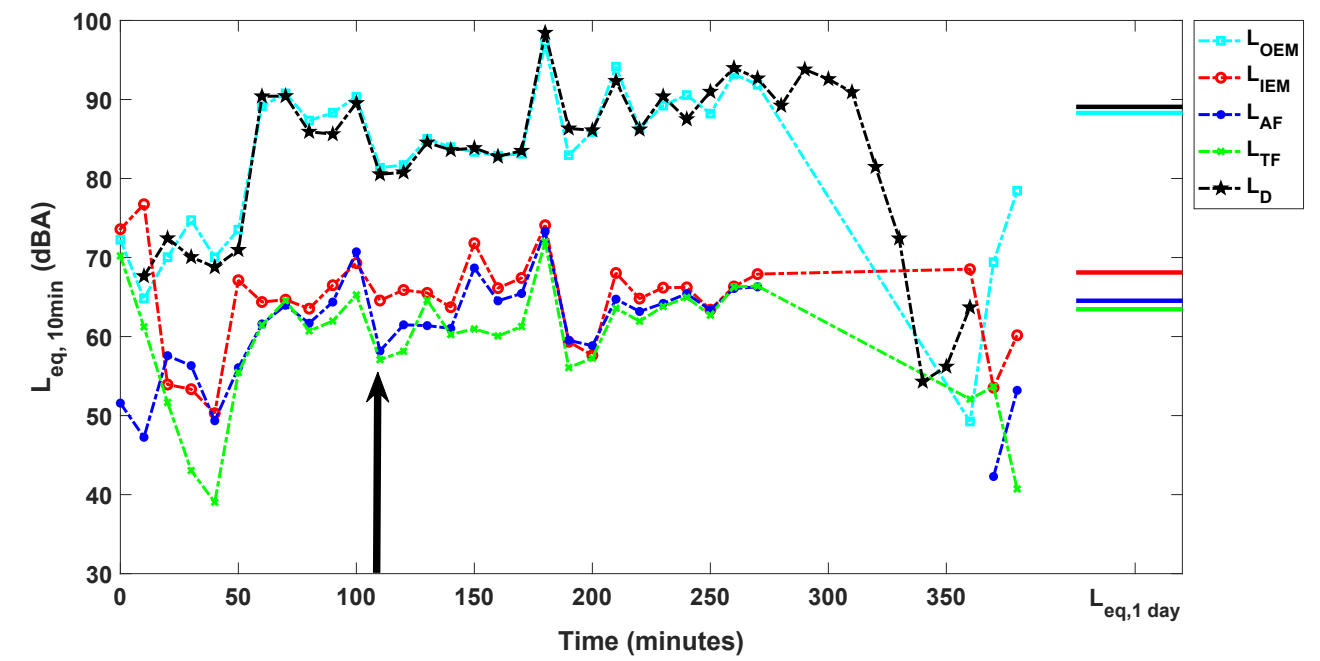

Figure 6: Example of typical cumulative equivalent noise levels, for the same individual as in Fig. 5. over one work day excluding $L_{\mathrm{AF}}$ when $\mathrm{PAR}_{\mathrm{AF}}<0$. The arrow indicates the index of the 10 minutes of the plot in Fig. 5b. Gaps in $L_{\mathrm{eq}}$ are explained by the device that was switched off, which was not the case of the reference dosimeter $\left(L_{D}\right)$

the adaptive filter, to estimate the WID-free residual SPL in any given situation. Indeed high-level WIDs, e.g. raised speech or shocks on the earpiece, may affect the noise levels measured by the OEM, therefore $L_{\mathrm{AF}}$ does not fully exclude WIDs, especially in low to moderate ambient SPLs. Hence, minor differences in the daily residual SPLs between the two methods are expected.

Another aspect to consider before using the proposed approach in commercial IENDs is the effects of the incoming noise directivity on the earplug transfer function. Preliminary measurements involving a subject wearing the earpieces used in this study showed that such transfer function could vary depending on the incidence angle of the sound source, especially at high frequencies. Up to $15.3 \mathrm{~dB}$ variations are observed between transfer function magnitudes estimated at $0,90,180$ and $270^{\circ}$ for frequencies above $4500 \mathrm{~Hz}$ in a $10 \mathrm{~m}^{2}$ sound booth with the source placed within $0.5 \mathrm{~m}$ from the subject's head. The impact of such variations on the estimated $L_{\text {eq }}$ should be assessed before using the proposed algorithm in free-field or when machinery with very localized noise sources are used in small rooms with low reverbera- 


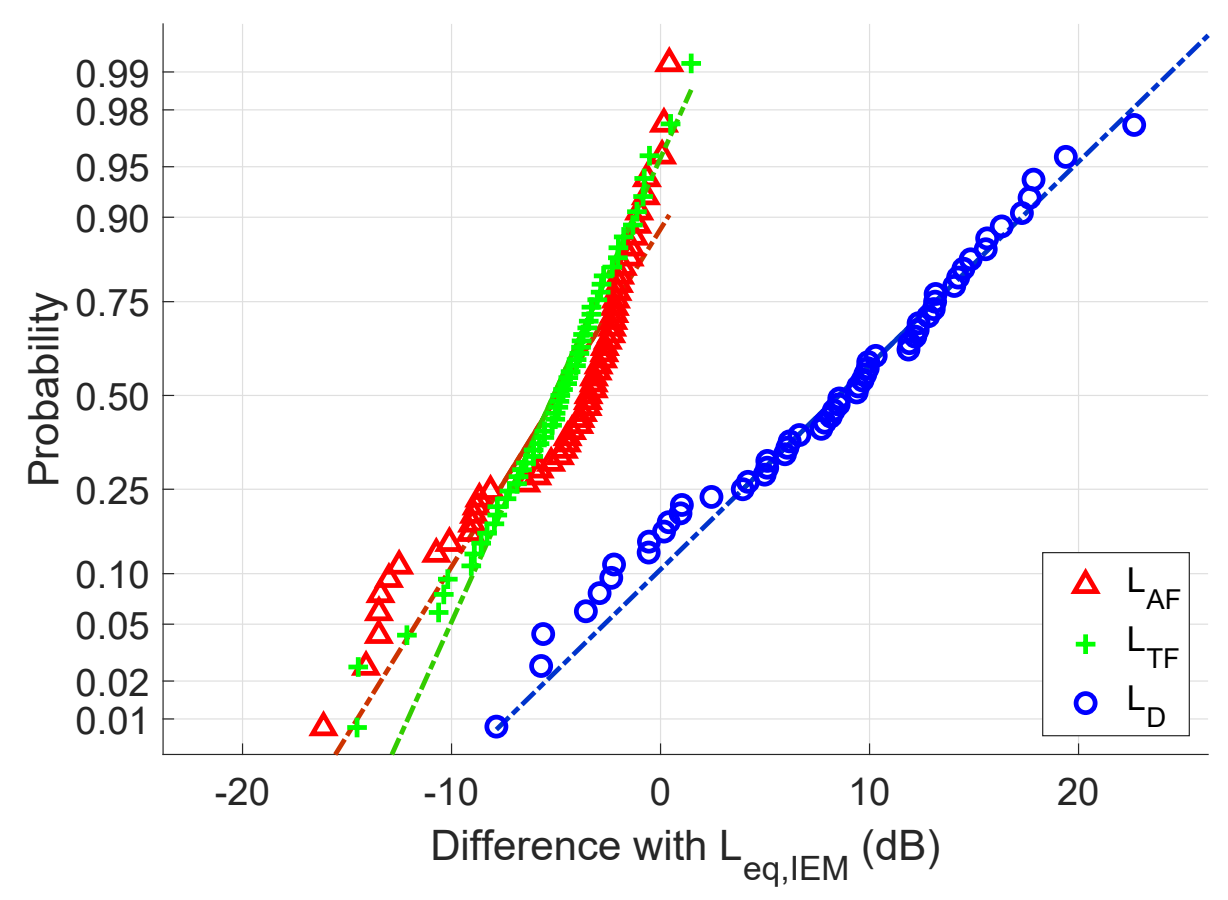

Figure 7: Normal probability plots showing the distribution of the IEM Equivalent sound level $\left(L_{\text {eq,IEM }}\right)$ differences accumulated on each day with the first proposed approach $\left(L_{\mathrm{AF}}\right)$ and the reference algorithm output $\left(L_{\mathrm{TF}}\right)$, where each data point is a test day, for every participant. The reference Larson Davis dosimeter $L_{\text {eq }}$ level $\left(L_{\mathrm{D}}\right)$ differences from the IEM are also included to show the important difference in daily accumulated levels between a microphone placed on the shoulder and a microphone placed in the earcanal 
tion time. Such differences in transfer functions could result in errors in the estimated $L_{\mathrm{eq}}$ and the PAR. However, the participants in the current study were only tested indoors where the effects of directivity are assumed to be minimal due to reverberation in the tested conditions. Moreover, the workers are moving continuously and noise sources can come from various tools surrounding them, hence logically the sum of the variations in the transfer function should result in a relatively small estimation error in the daily $L_{\text {eq }}$.

The adaptive filtering approach proposed in this paper is an interesting alternative to the reference method used for comparison [26, 24]. Although this new method may occasionally overestimate the in-ear noise exposure due to the influence of high-level WIDs (e.g. raised speech) on the OEM, it would allow to better account for the ambient SPL variations occurring during such WIDs and hence be more adapted to highly fluctuating noise environments. The low complexity of the proposed algorithm also requires less computational power, this results in a computation time of approximately 0.3 and 0.005 s faster to calculate the residual SPL and PAR respectively, compared to the reference methods as measured using MATLAB. These differences are mostly explained by the elaborate code for the decision process of the reference WID rejection algorithm and the extra calculation step to calculate the PAR. Hence, the proposed algorithm is a good option for implementation in future commercial IEND devices. Nevertheless, this method may be less adapted to low noise environments as it requires a good identification of the earplug's transfer function in the individual's ear, before WIDs are detected. It also requires a sufficiently high ambient noise level to correctly reject the WIDs and estimate the PAR.

As mentioned earlier, to prevent the proposed approach's filter of adapting during WIDs, which leads to an underestimation of the earplug transfer function and an overestimation of the residual SPL, the earplug transfer function can also be identified at an optimal moment during the day. For example, when the noise level is sufficiently high and the wearer is quiet, since swallowing is sufficient to generate an in-ear SPL of approximately 65 dBA [24] and could lead to a negative PAR. Using the data collected in this study, this scenario was simulated on a typical subject and lead to an additional residual SPL reduction of approximately $1.3 \mathrm{~dB}$ when compared to the 30-second filter coefficients update, matching the reference algorithm's $L_{\mathrm{TF}}$ more closely. Changing the filter adaptation interval to 2-minute and 10-minute resulted in negligible changes compared to the residual SPLs obtained with the $30 \mathrm{~s}$ interval. Hence, adapting the filter every $30 \mathrm{~s}$ is a good 
compromise between continuously adapting the filter to track changes in the transfer function due to head movement and reducing the risk of adapting the filter while a WID is present in the signal. Ideally, the earplug transfer function should be updated regularly at a short-time interval, to ensure that potential changes in the earplug's fit are considered in the estimated in-ear noise level. For instance, the user could trigger an adaptation at regular time intervals whenever the dosimeter displays ambient noise levels higher than a certain threshold value to be defined. The user would have to remain quiet during the adaptation to obtain an accurate earplug transfer function. As a further verification step, the estimate could be discarded if too much variation is found in the PAR during adaptation.

The two algorithms were found to lower the estimated in-ear noise exposure due to WID rejection by 3.4 and $4.8 \mathrm{~dB}$ for the proposed and reference algorithms respectively, as shown in Fig. 7, when most participants were exposed to daily ambient SPLs varying from approximately 80 to $90 \mathrm{dBA}$. Less reduction in residual SPL would be expected from WID rejection in higher ambient noise levels as the WIDs would have less impact on the residual SPL.

The reduction of the WIDs' impact in the accumulated noise exposure levels can be important for occupational health purposes as the inclusion or rejection of WIDs could double or halve an individual's daily dose over the same time duration, when an exchange rate of $3 \mathrm{~dB}$ is considered, in function of the ambient noise level. Moreover, it is clear that a shoulder microphone $\left(L_{D}\right)$ overestimates $(9.4 \mathrm{~dB})$ the actual noise exposure that can affect the hearing health of a protected individual. Whereas the negative $L_{\mathrm{D}}$ differences shown in Fig. 7, such as $-7.9 \mathrm{~dB}$, are obtained from participants exposed to low ambient noise levels (ranging from approximately 60 to $70 \mathrm{dBA}$ at the OEM) on specific days. At such levels, physiological noises (breathing, swallowing, chewing, etc.) and low-level speech contribute to the in-ear SPLs while having a negligible effect on the shoulder microphone. Therefore, the in-ear residual SPL estimated with proper earplug transfer function, microphone-to-eardrum corrections and WID rejection [35, 24, 26] should be considered to assess the risk of NIHL with the effective noise dose at the eardrum position in future studies.

\section{Conclusions}

A low-complexity method using adaptive filtering is proposed to detect wearer-induced disturbances and reject them from the residual SPL in the 
ear. This method is able to subtract the impact of WIDs in moderate and high ambient SPLs and performs well with quick changes in ambient noise levels during WIDs. The performance of the algorithm in low ambient SPLs requires a good earplug transfer function identified a-priori, otherwise an incorrect residual SPL is estimated. This method could eventually be integrated in IEND systems to run in real-time to estimate the residual SPL behind the earplug and the hearing protection level instantaneously using the proposed PAR estimate to provide users important information on their level of protection and daily in-field noise levels.

The performance of the proposed method is compared to a reference algorithm which achieves more WID rejection in residual SPL for low ambient SPL conditions. Both the proposed and reference methods could lead to a lower 1-day cumulative noise level by rejecting the WIDs and such reduction could more than double the allowed noise exposure duration for an exchange rate of $3 \mathrm{~dB}$. The integration of such methods in a portable system could eventually help understand the relationship between the noise levels inside the ear and their effects on hearing health, especially if it is combined with otoacoustic emission measurements [36]. In the long run, it may also help assess the effects of WIDS, e.g. speech, on hearing health.

\section{Acknowledgment}

The authors would like to acknowledge the financial support received from the Natural Sciences and Engineering Research Council (NSERC) through the NSERC-EERS Industrial Research Chair in In-Ear Technology (CRITIAS), as well as for technical support provided by EERS Global Technologies Inc. The authors would also like to thank the Centre for Interdisciplinary Research in Music Media and Technology (CIRMMT) for project funding opportunities. The first author is grateful to the Institut de recherche Robert-Sauvé en santé et sécurité du travail (IRSST) for the financial support received during his doctoral program and to the École nationale du meuble et d'ébénisterie (ENME) and its students for their participation in the field data collection.

\section{References}

[1] P. M. Rabinowitz, D. Galusha, C. Dixon-Ernst, J. E. Clougherty, and R. L. Neitzel, "The dose-response relationship between in-ear occupational noise exposure and hearing loss.," Occupational and environmental medicine, vol. 70, no. 10, pp. 716-21, 2013. 
[2] NIOSH, "National Institute for Occupational Safety and Health : NOISE AND HEARING LOSS PREVENTION." https://www.cdc. gov/niosh/topics/noise/, May 2019.

[3] K. Feder, D. Michaud, J. McNamee, E. Fitzpatrick, H. Davies, and T. Leroux, "Prevalence of hazardous occupational noise exposure, hearing loss, and hearing protection usage among a representative sample of working canadians," Journal of occupational and environmental medicine, vol. 59, no. 1, p. 92, 2017.

[4] K. Tantranont and N. Codchanak, "Predictors of hearing protection use among industrial workers," Workplace health 85 safety, vol. 65, no. 8, pp. 365-371, 2017.

[5] E. H. Berger, J. R. Franks, A. Behar, J. G. Casali, C. Dixon-Ernst, R. W. Kieper, C. J. Merry, B. T. Mozo, C. W. Nixon, D. Ohlin, et al., "Development of a new standard laboratory protocol for estimating the field attenuation of hearing protection devices. part iii. the validity of using subject-fit data," The journal of the acoustical society of america, vol. 103, no. 2, pp. 665-672, 1998.

[6] P. S. Smith, B. A. Monaco, and S. L. Lusk, "Attitudes toward use of hearing protection devices and effects of an intervention on fit-testing results," Workplace health Ef safety, vol. 62, no. 12, pp. 491-499, 2014.

[7] M. R. Groenewold, E. A. Masterson, C. L. Themann, and R. R. Davis, "Do hearing protectors protect hearing?," American journal of industrial medicine, vol. 57, no. 9, pp. 1001-1010, 2014.

[8] HSE, "Health and safety executive : Removal of hearing protectors severely reduces protection." http://www.hse.gov.uk/noise/ hearingprotection/index.htm/, May 2019.

[9] M. Borgh, F. Lindström, K. P. Waye, and I. Claesson, "The effect of own voice on noise dosimeter measurements: a field study in a day-care environment, including adults and children," in Internoise, 2008.

[10] S. Mukerji, A. M. Windsor, and D. J. Lee, "Auditory brainstem circuits that mediate the middle ear muscle reflex," Trends in amplification, vol. 14, no. 3, pp. 170-191, 2010. 
[11] O. Creutzfeldt, G. Ojemann, and E. Lettich, "Neuronal activity in the human lateral temporal lobe," Experimental Brain Research, vol. 77, no. 3, pp. 451-475, 1989.

[12] ANSI, "Methods for measuring the real-ear attenuation of hearing protectors," 2008.

[13] R. Neitzel, S. Somers, and N. Seixas, "Variability of real-world hearing protector attenuation measurements," The Annals of occupational hygiene, vol. 50, no. 7, pp. 679-691, 2006.

[14] H. Nélisse, M.-A. Gaudreau, J. Boutin, J. Voix, and F. Laville, "Measurement of hearing protection devices performance in the workplace during full-shift working operations," Annals of occupational hygiene, vol. 56, no. 2, pp. 221-232, 2011.

[15] E. Berger, "Is real-ear attenuation at threshold a function of hearing level?," The Journal of the Acoustical Society of America, vol. 78, no. 5, pp. 1588-1595, 1985.

[16] J. Voix, Smith, Pegeen, and Berger, Elliott, "Field Fit-Testing and Attenuation Measurement Procedures," in The Noise Manual, Am. Ind. Hyg. Assoc. J., 6 ed., 2019. In press.

[17] 3M, "What is hearing protection fit testing?" https://safetytownsquare.3mcanada.ca/articles/ what-is-hearing-protection-fit-testing, February 2020.

[18] E. H. Berger, "What is a Personal Attenuation Rating (PAR)?," Tech. Rep. 2.31, 3M Occupational Health \& Environmental Safety Division, 3M Occupational Health \& Environmental Safety Division E•A $\bullet$ RCAL Laboratory, Apr. 2010.

[19] F. Bonnet, J. Voix, and H. Nélisse, "The opportunities and challenges of in-ear noise dosimetry," Canadian Acoustics, vol. 43, no. 3, 2015.

[20] M. A. Theis, H. L. Gallagher, R. L. McKinley, and V. S. Bjorn, "Hearing Protection With Integrated In-Ear Dosimetry: A Noise Dose Study," in ASME 2012 Noise Control and Acoustics Division Conference, (New York City, New York, USA), p. 237, ASME, Aug. 2012. 
[21] S. K. Davis, P. T. Calamia, W. J. Murphy, and C. J. Smalt, "In-ear and on-body measurements of impulse-noise exposure," International journal of audiology, vol. 58, no. sup1, pp. S49-S57, 2019.

[22] C. J. Smalt, J. Lacirignola, S. K. Davis, P. T. Calamia, and P. P. Collins, "Noise dosimetry for tactical environments," Hearing research, vol. 349, pp. 42-54, 2017.

[23] J. A. Trawick, J. Slagley, and R. M. Eninger, "Occupational Noise Dose Reduction via Behavior Modification Using In-Ear Dosimetry among United States Air Force Personnel Exposed to Continuous and Impulse Noise," 2019.

[24] F. Bonnet, H. Nélisse, M. A. Nogarolli, and J. Voix, "In-ear noise dosimetry under earplug: Method to exclude wearer-induced disturbances," International Journal of Industrial Ergonomics, vol. 74, p. 102862, 2019.

[25] E. Berger and J. Kerivan, "Influence of physiological noise and the occlusion effect on the measurement of real-ear attenuation at threshold," The Journal of the Acoustical Society of America, vol. 74, no. 1, pp. 81-94, 1983.

[26] F. Bonnet, Méthode de mesure individuelle de l'exposition sonore effective intra-auriculaire en milieu de travail. $\mathrm{PhD}$ thesis, École de technologie supérieure, Montréal (Canada), 2019.

[27] J. Voix, Mise au point d'un bouchon d'oreille" intelligent". PhD thesis, École de technologie supérieure, 2006.

[28] R. B. Randall and B. Tech, Frequency analysis. Brüel \& Kjær, 1987.

[29] E. H. Berger, The noise manual. Aiha, 2003.

[30] CRITIAS, "NSERC-EERS Industrial Research Chair in In-Ear Technologies (CRITIAS) - Auditory Research Platform (ARP)," 2019.

[31] V. Nadon, A. Bockstael, D. Botteldooren, and J. Voix, "Assessment of otoacoustic emission probe fit at the workfloor," in 10th European Congress and Exposition on Noise Control Engineering (Euronoise 2015), pp. 1955-1960, 2015. 
[32] V. Nadon, A. Bockstael, D. Botteldooren, J.-M. Lina, and J. Voix, "Design considerations for robust noise rejection in otoacoustic emissions measured in-field using adaptive filtering," Acta Acustica united with Acustica, vol. 103, no. 2, pp. 299-310, 2017.

[33] F. Bonnet, H. Nélisse, and M. A. C. Nogarolli, "An in-ear noise dosimetery method that excludes the sounds generated by individuals wearing earplugs: preliminary field study," in International Congress on Sound and Vibration, 2019.

[34] ISO, "Acoustics - Determination of sound immission from sound sources placed close to the ear - Part 1: Technique using a microphone in a real ear (MIRE technique)," 2002.

[35] F. Bonnet, H. Nélisse, M. A. Nogarolli, and J. Voix, "Individual in situ calibration of in-ear noise dosimeters," Applied Acoustics, vol. 157, p. 107015,2020 .

[36] V. Nadon, A. Bockstael, D. Botteldooren, and J. Voix, "Field monitoring of otoacoustic emissions during noise exposure: Pilot study in controlled environment," American journal of audiology, vol. 26, no. 3S, pp. 352$368,2017$. 